\title{
A Mini-Review of Nanotechnology and Prostate Cancer: Approaches in Early Diagnosis
}

\section{Marie Saghaeian Jazi ${ }^{1,2 *}$}

1. Metabolic Disorders Research Center, Golestan University of Medical Sciences, Gorgan, Iran.Orcid: 0000-0003- 0647-9545. Email: marie.saghaeian@goums.ac.ir.

2. Stem Cell Research Center, Golestan University of Medical Sciences, Gorgan, Iran

*Correspondence: Marie Saghaeian Jazi, Stem Cell Research Center, Golestan University of Medical Sciences, Gorgan, Iran Tel:+1732455166 Email:

marie.saghaeian@goums.ac.ir.

Received Mar 14,2020;Accepted Apr 23,2020

\begin{abstract}
The most important aspect of cancer treatment is early diagnosis. The best serum marker currently available for diagnosis of prostate cancer $(\mathrm{CaP})$ is serum prostate-specific antigen (PSA). However, PSA test does not have high specificity and is not reliable for differentiating benign prostate hyperplasia, non-aggressive $\mathrm{CaP}$ and aggressive $\mathrm{CaP}$. In the past decade, great efforts have been made in the development of novel biosensor-based strategies for detection of biomolecules and miniaturization assays for PSA. The emerging nanotechnology in recent years is expected to have a profound effect on healthcare and scientific research in the near future. Specifically, nanotechnology is foreseen to help solve one of the most challenging and longstanding problems of early cancer detection. The current mini-review summarizes the current knowledge and application of nanoarrays, nanosensors, liposomes, improved nanoparticles (dendrimers, diamondoids, gold-based nanoparticles, magnetic nanoparticles and quantum dots) and nanoelectronics in early diagnosis of prostate cancer. This mini-review highlights the most recent advances and innovative solutions in applications of nanotechnology for the detection of $\mathrm{CaP}$ biomarkers and early diagnosis of $\mathrm{CaP}$.
\end{abstract}

Keywords: Prostate cancer, Nanotechnology, Diagnosis

Article Type: Review Article 


\section{INTRODUCTION}

Prostate cancer is the second most common cancer and the fifth cause of cancer death in men (1). Prostate specific antigen (PSA) is the most widely used tumor marker for diagnosis of prostate cancer $(\mathrm{CaP})$. Elevated amounts of this biomarker in blood serum (more than $4 \mathrm{ng} / \mathrm{ml}$ ) can be a sign of cancer (2). The importance of early detection in all cancers as well as in $\mathrm{CaP}$ is obvious; due to the fact that at early stages of cancer, treatment will be more effective (3). Nanotechnology can present unique approaches in cancer detection techniques and consequently, many efforts have been made to develop novel nanotechnology-based diagnostic tools to detect cancer at early stages (4). With the help of these tools, it would be possible to detect the smallest concentrations of PSA (or any other $\mathrm{CaP}$ biomarker). There are different nanoparticlebased molecular methods for early detection of $\mathrm{CaP}$, which can be categorized in two main types of label free and label based (Table 1). Each method has some advantages with varied detection limits; however, all of them can potentially improve the current knowledge of $\mathrm{CaP}$ early detection.

The focus of this mini-review is to summarize nano-based $\mathrm{CaP}$ detection techniques currently in use or at research stage. First, the known biomarkers related to $\mathrm{CaP}$ detection will be discussed and then the latest nanotechnology-based $\mathrm{CaP}$ detection techniques will be introduced.

Table 1. Summary of different methods for CaP detection

\begin{tabular}{|c|c|}
\hline Categories & Method (examples) \\
\hline \multirow{2}{*}{$\begin{array}{c}\text { Label-based nano- } \\
\text { biosensing }\end{array}$} & Fluorescent nanoparticles (Quantum Dot, Europium, Terbium Complex) \\
\cline { 2 - 2 } & Magnetic nanoparticles (Gold nanoparticle) \\
\cline { 2 - 2 } & Surface enhanced raman scattering (SERS) nanoparticles \\
\hline $\begin{array}{c}\text { Label-free nano- } \\
\text { biosensing }\end{array}$ & $\begin{array}{c}\text { Nano wire, Surface plasma resonance (SPR), Carbone nanotube, electrical } \\
\text { biosensors, microcantilvers, Scanning tunneling microscopy (STM) }\end{array}$ \\
\hline
\end{tabular}

\section{Prostate cancer biomarkers}

In cell biology, a biomarker is defined as an agent that helps in the detection and isolation of a particular cell type. In medicine, a biomarker can be any substance that could be used for examining an organ function or other aspects of health (5). It can also be a biomolecule whose detection indicates a particular disease state, for example, the presence of PSA in blood serum, higher than a specific level can be sign of prostate hyperplasia or cancer (6-9). Different $\mathrm{CaP}$ biomarkers have been developed in recent years (Table 2). These biomarkers can be used in diagnosis tests and monitoring disease progression (9). Despite all the progress made throughout the years in developing new $\mathrm{CaP}$ biomarkers, $\mathrm{CaP}$ is still one of the most challenging cancers in men. Early, specific diagnosis is an important aspect of cancer treatment. The biomarkers presented bellow pose unique characteristics as well as some disadvantages. Hence, it is believed that no individual biomarker is ideal. The most sensitive, specific diagnostic tests would be those able to detect a combination of all these markers and this is where nanotechnology can be very useful. 
Table 2. Summary of important CaP biomarkers

\begin{tabular}{|c|c|c|c|c|}
\hline Biomarker & Type & Localization * & $\begin{array}{c}\text { Gene } \\
\text { name }\end{array}$ & GO term** (Biological process) \\
\hline $\begin{array}{c}\text { Prostate specific antigen / } \\
\text { Human kallikerin 3 }\end{array}$ & Protein & Secreted & KLK3 & Proteolysis (GO:0006508) \\
\hline Prostate cancer gene 3 & ncRNA & Extracellular & PCA3 & - \\
\hline Prostate stem cell antigen & protein & $\begin{array}{c}\text { Plasma } \\
\text { membrane }\end{array}$ & PSCA & $\begin{array}{c}\text { negative regulation of ERK1 and ERK2 } \\
\text { cascade (GO:0070373) }\end{array}$ \\
\hline $\begin{array}{c}\text { Prostate-specific membrane } \\
\text { antigen }\end{array}$ & Protein & $\begin{array}{c}\text { Plasma } \\
\text { membrane }\end{array}$ & FOLH1 & $\begin{array}{c}\text { C-terminal protein deglutamylation } \\
\text { (GO:0035609) }\end{array}$ \\
\hline
\end{tabular}

*Adopted from UniPortKB and GeneCard database

**Adopted from QuickGO

Prostate specific antigen, also known as the human kallikerin $3(\mathrm{hk} 3)$ is a $33 \mathrm{kD}$ glycoprotein and a member of the family of human kallikerin proteins. It is also recognized as a neutral serine protease $(6,10$, 11). It is secreted by pancreatic ducts, the prostatic epithelium and the epithelial lining of the acini $(9,12)$. When entering the circulatory system, PSA is quickly trapped by protease inhibitors, primarily 1antichymotrypsin (ACT). However, an amount of it is cleaved by proteases and circulates in the lumen in its inactive form, free PSA (f-PSA). The complex of f-PSA and PSA/ACT is referred to as T-PSA (total PSA), which rises in serum during $\mathrm{CaP}$. Hence, T-PSA monitoring can help in diagnosis of $\mathrm{CaP}$. Prostate specific antigen usually exists in male serum at concentration of around $1 \mathrm{ng} / \mathrm{ml}$, and its accepted normal cut-off value is $4 \mathrm{ng} / \mathrm{ml}$ (although there can be some age-specific augmentations). It should be noted that the cut-off limit of T-PSA between prostate hyperplasia and cancer is 4 $\mathrm{ng} / \mathrm{ml}$, and concentration of $4-10 \mathrm{ng} / \mathrm{ml}$ is considered as "diagnostic gray zone", which predicts the possibility of prostate carcinoma $(6,7,10)$.

Different studies have demonstrated that various factors such as tumor mass, presence or degree of prostatic inflammation, circulating levels of growth factors and even presence of chemo-preventers can alter the serum PSA level, indicating the capacity of PSA as an ideal biomarker, not only in prostate disease, but also in tracing the state of progress $(7,13)$.

The human kallikerin $2(\mathrm{hk} 2)$ is a member of the serine protease family with $80 \%$ amino acid sequence homology to that of PSA. The kallikeringene family has 15 members and the most important member of this family after hk3 (PSA) is hk2. Beside these, hk 4, 5, 8, 9, $10,11,13,14$ and 15 have been reported as novel biomarkers for detection of prostate, ovarian and breast cancers (12). The presence of kallilkerin in free and bound forms, in blood, has been frequently reported (9). The overexpression of $\mathrm{hk} 2$ in $\mathrm{CaP}$ was initially detected by immunohistochemical staining, and later confirmed by other investigations, not only in $\mathrm{CaP}$, but also in prostatic intraepithelial neoplasia $(7,9)$. It is believed that co-usage of PSA and hk2 as biomarkers can improve the accuracy of $\mathrm{CaP}$ diagnosis tests (7).

Prostate cancer gene 3 (PCA3), also known as DD3PCA3 or PCA3DD3, is another known marker for $\mathrm{CaP}$ detection. It is a non-coding specifically overexpressed in most $\mathrm{CaP}$ specimens from the early stages. It was initially found by differential display analysis, a test in which mRNAs expressed in normal and tumor-bearing prostate tissues are compared. According to clinical studies, more accurate results compared to PSA-based tests can be achieved by choosing PCA3 as a biomarker $(9,12)$. The notable disadvantage of this biomarker is that urine testing requires prostate massage; in order to force the cells, especially tumor cells, to enter the prostatic 
urethra, which can result in some unwanted variation in total biomarker release, because of the differences in duration and intensity of the massage between patients. Therefore, the whole test in general is not efficient enough for clinical use (12).

Prostate stem cell antigen (PSCA) is an integral glycosylphosphatidylinositolanchored protein that exists on the outer surface of the membrane. It is significantly overexpressed during prostatic intraepithelial neoplasia and $\mathrm{CaP}$, making PSCA a great biomarker for diagnosis of prostatic diseases (14). This marker is another prostate epithelium membrane antigen whose expression rises during $\mathrm{CaP}$ stages. It is highly expressed in androgen-independent cancer cells. However, its low expression in normal prostate, kidney, brain and small intestine tissues has been reported. The advantage of this marker is that the level of PSMA could discern between late and early stages of $\mathrm{CaP}(9,15)$.

\section{Label-based nanobiosensing}

Different and variable labels can be used in combination with nanotechnology for cancer detection. One of the most common methods is to use fluorescent properties for labeling. Quantum dots (QDs) are inorganic flurophores ranging from $2-10 \mathrm{~nm}$ in size. The size of the QD can determine its emission wavelength and color. Several features, such as their size variation, high levels of brightness and photo stability make QDs perfect tools in imaging and biological detection (16-19). These structures can be easily excited by a single light source, even white light. Other excitation sources are UV light, blue-violet filtered light and 405 and $488 \mathrm{~nm}$ lasers (16). On the other hand, QDs are much more resistant to photobleaching and are around 50 times brighter in comparison with fluorescent proteins and organic dyes (19). In an experience, Dong et al. have used water soluble CdTe QDs for $\mathrm{CaP}$ cells' detection. The CdTe QDs were initially coated by L-glutathione and then conjugated with mouse anti-human PSA antibody to directly detect $\mathrm{CaP}$ cells.
Meanwhile, goat anti-mouse IgG was linked to CdTe QDs for indirect detection. The results showed that both direct and indirect labeling have strong fluorescence intensity (17). In another case study, Härmä et al. used $107 \mathrm{~nm}$ streptavidin-coated QDs including $\beta$ diketones entrapping N 30,000 europium molecules to detect PSA in mice and reported in vivo detection of $\mathrm{CaP}$ xenografts using antibody-coupled QDs (16). |In general, antibody-linked QDs can be used as great probes in cell imaging and cancer cells' detection $(17,18)$.

More recently, aptamer-conjugated QDs were developed to target membrane PSA, which can be applied in both imaging and smart drug delivery. Using aptamer conjugated CdSe/ZnS QD 490 (emission: 470-530 nm which overlaps excitation of doxorubicin $\sim 480 \mathrm{~nm}$ ), it is possible to use fluorescence resonance energy transfer for imaging and targeted drug delivery sensing. The cited simultaneous imaging, therapy and sensing system has worked successfully in vitro (20). Recently, it has been shown that bioconjugated near infrared (NIR) QD probes can improve imaging and visualization of tumors, especially in deep tissues $(21,22)$. In order to prevent degradation of the QDs, specific modifications such as addition of amphiphilic triblock co-polymers (containing hydrophilic polymethacrylic segments, two hydrophobic segments of polybutylacrylate and polyethylacrylate) have been suggested. To improve bioavailability, biophile polymers like polyethylene glycol molecules can be added to QDs. In a case study, metastatic CaP cells in mouse bone (tibia) were successfully detected by PSMA antibody-conjugated NIR QDs. The detection limit of this technique was 500,000 prostate cells $(0.5 \mathrm{mg}$ of tumor mass) in mouse tibia (21).

Another label used for $\mathrm{CaP}$ detection is europium (III), which has been widely used as a nanoparticle, partly because of its long lasting fluorescent property. Streptavidincoated and antibody conjugated europium (III) nanoparticles are good, modern detectors both in solid and liquid phases. Several studies have used this complex for the 
detection of various cell markers, including PSA $(23,24)$.

Fluorescent terbium nanoparticles are also handy materials that can be applied in bioassays and cancer diagnosis tests, partly because of their long lasting fluorescence lifetime. With either surface modifications or bioconjugation of antibodies or streptoavidin, terbium nanoparticles can become great, sensitive detectors. In addition, non-toxicity and high stability in basic solutions make these materials more beneficial in various conditions compared to other nanoparticles such as silica-based ones (25).

As a good replacement for fluorescence labeling, surface-enhanced Raman scattering (SERS) is a common technique used in biosensor development. It is used to investigate the vibrational properties of adsorbed molecules by the help of both visible and NIR light. In addition, SERS immunoassays based on antigen-antibody interactions have been widely used in development of biomarkers for early cancer diagnosis. In such assays, Raman dyes are used to label antibodies attached to the nanoparticle probe's surface; the light scattered from the Raman reporter molecule or RRM - which is usually a metal nanoparticle who gets excited in Raman spectroscopy- provides information about the vibrational quantum states of the molecule (2, 26, 27). Compared to fluorescent labels, SERS has some unique advantages: it produces sharper, more specific bands (10100 times narrower), the excitation wavelength required for SERS is a function of size and composition of the nanoparticle, it could be used in a variety of environments for it is not sensitive to oxygen or humidity, it is less susceptible to photobleaching and has lower limit of detection (28). All these characteristics have made SERS an appropriate, modern diagnostic technique in cancer detection (29-31). Reports have been given on the usage of SERS in a sandwichimmunoassay format using antigen-antibody interactions for the detection of very low concentrations $(1 \mathrm{pg} / \mathrm{ml})$ of $\operatorname{PSA}(2,27,29)$. Another bioanalytical application of SERS is composite organic-inorganic nanoparticles, which are composed by aggregating $\mathrm{Ag}$ nanoparticles in the presence of Raman reporter molecules. Husdon and Chumanov used the same technique for in vitro detection of antigen in tissue specimens. They also managed to detect PSA in situ in epithelial prostate tissue (30). In another experiment, Cheng Sun and colleagues used nanoplasmonic resonators (NPRs) for sensitive detection of protease activity, including proteolytically active PSA, in realtime. This, this can be considered as a fast, sensitive and specific technique for one-step detection of proteases activities in very small samples (31).

Magnetic nanoparticles (MNPs) are generally derived from magnetic elements (iron, nickel, cobalt and their oxides), so they can be concurrently functionalized and directed by a magnetic field (32). Super paramagnetic iron oxide (SPIO) is a nanoprobe used in cancer detection. Features such as magnetic resonance signal sensitivity, great MRI T2 contrast, low detection limit, low toxicity and excellent biocompatibility has made SPIO an ideal marker in magnetic resonance imaging (MRI). In an in vitro study, the PEG-g-PEI-SPIO complex with a single antibody (scAbPSCA) has helped decrease the MRI T2 signal intensity of $\mathrm{CaP}$ cells, resulting in improved imaging effects (14).

Beside early detection, magnetic nanoparticles have attracted a lot of attention as modern cancer therapy equipments. In thermotherapy, the temperature should reach up to $42 \square \mathrm{C}$, while maximum temperature of $55 \square \mathrm{C}$ could be achieved in the prostate tissue thermotherapy by the help of MNPs (33).

Gold nanoparticles (AuNP) have great affinity to leaky tumor vasculature (which has been shown in tumor angiogenesis during tumor growth) and unique tumor retention capabilities. In the last decade, usage of gold 
nanoparticles in radio-sensitization of cancer cells, such as CaP cells has been reported (34, 35). Moreover, AuNPs are used as catalysts, for signal amplification in organic and electrochemical reactions. Das and colleagues have used AuNPs as catalysts for signal amplification of p-nitrophenol reduction to $\mathrm{p}$ aminophenol. They reported the detection limit of $1 \mathrm{fg} / \mathrm{ml}$ for PSA (36). Moreover, different substances such as GA, a plant extract which is used as a food additive, can be used for increasing gold nanoparticles' stability under both in vivo and in vitro conditions. This biomolecule strongly binds to AuNPs on the protein matrix by the help of its glycoprotein backbone and creates a nontoxic nano-sized complex. The effectiveness of this technique has been shown in radiotherapy and prostate tumor regression without affecting non-target organs $(34,37)$. Using biobarcodes for nanoparticle fictionalization can help the sensitivity and specificity of CaP detection. Barcode DNA functionalized AuNPs for PSA are 300 times more sensitive than common immunoassays (38).

More recently, Xia et al. developed a colorimetric assay based on AuNPs coated with PSA peptide with a detection limit of $0.02 \mathrm{ng} / \mathrm{mL}$ for PSA (39).

\section{Label free nanobiosensing}

Nanowires are known for their high surfaceto-volume ratio and high electronic conductance, which can be simply influenced by any surface perturbations, such as binding of macromolecules (40). By using silicon nanowires, Lieber et al. introduced a labelfree, multiplexed method of PSA, PSAalphal-antichymotrypsin,

carcinoembryonicantigen and mucin- $\mathrm{L}$ detection (41). The immunological detection of T-PSA using n-type Ln2O3 nanowires and p-type carbon nanotubes on a field-effect transistor-based device has been also reported by Lin and colleagues $(29,30)$.

Nanotubes, especially CNTs are considered as one of the most promising candidates for nano-sized biosensors because of their electrical and mechanical characteristics. For example, label-free amperometric immunosensors, with CNT electrodes anchored to PSA-mAb, have been used for detection of PSA between the range of 0.25-1 $\mathrm{ng} / \mathrm{ml}$. Since prostate hyperplasia and cancer have a PSA concentration difference of 4 $\mathrm{ng} / \mathrm{ml}$, the cited electrochemical immunosensor could be beneficial in clinical detections (42). In another case study using the mean of differential pulse voltammetry, TPSA was successfully detected using a SWNT-modified microelectrode immunoassays with detection limit equal to $0.25 \mathrm{ng} / \mathrm{ml}$, which is more than the cut-off limit of T-PSA between hyperplasia and $\mathrm{CaP}$ (43). $\mathrm{Yu}$ and colleagues have fabricated SWNT forest platforms filled with multilabel, secondary antibody-SWNT bioconjugates for sensitive electrochemical immune detection of PSA in serum and tissue lysates (10). Gold modified carbon nanotubes can electrochemically measure PSA in only five minutes, with detection limit of $1 \mathrm{ng} / \mathrm{ml}$ (44).

The antibody-functionalized Au-gated AlGaN/GaN high electron mobility transistors (AlGaN/GaN HEMTs) are capable of detecting PSA at concentrations ranging between $10 \mathrm{pg} / \mathrm{ml}$ to $1 \mu \mathrm{g} / \mathrm{ml}$. Since its minimum detectable concentration is even less than the cut-off value of PSA for clinical detection, the AlGaN/GaN HEMTs seem to be appropriate electrical biosensors in early cancer diagnosis (45). Jae-HyukAhn et al. have created a field effect transistor device that can detect PSA without much labeling process. In this device, anti-PSA molecules are immobilized on the surface of a molecular-sized nanogap, which is the location of marker (PSA) absorption. This absorption can change the threshold voltage, which can allows electrical detection of the specific binding of PSA (46).

Surface plasmon resonance (SPR) is an affinity-based optical detection technique that involves the interaction of light with electrons of a material, such as a metal $(2,47)$. This technology has advantages such as low sample requirement and real-time, rapid and 
label-free monitoring of biomolecular reactions $(2,48)$. However, one of the disadvantages of SPR is its low sensitivity. The specific binding of a biomarker such as PSA to its antibody can be monitored by using local SPR that allows detection of PSA concentrations as low as $0.1 \mathrm{pg} / \mathrm{ml}(49-51)$.

The topological and electrical differences caused by attraction of nanoparticle conjugated antibodies on a specific surface containing PSA antigen can be observed by label free scanning tunneling microscopy (41), which is capable of ultrasensitive electrical detection of PSA concentrations as low as $10 \mathrm{fg} / \mathrm{mL}$ has (52).

Microcantilevers can be used as chemical, physical or biological sensors by detecting changes in cantilever bending or vibrational frequencies $(53,54)$. Recently, nanocantilever sensors have been developed for detection of $\mathrm{CaP}$ tumor-associated antigens $(55,56)$.

\section{Future perspectives and conclusion}

Lifestyle changes, environmental pollutants and an increase in life expectancy are among the major causes of cancer throughout the world. Prevention and early detection of cancer are major health issues in many countries. Although molecular targeted biomarkers have dramatically improved cancer diagnosis in the past two decades, with a significant impact on the diagnosis and staging of $\mathrm{CaP}$, development of new methods and new strategies is still essential.

Application of nanotechnology in molecular diagnostics is still in early stages; however, the application of nanotechnology and development of nanoparticle-based cancer diagnostics in medicine have experienced a rapid growth. It is believed that efficient, specific diagnosis of $\mathrm{CaP}$ is possible via tests that are able to detect multiple biomarkers. On the other hand, while PSA is now the most commonly used biomarker in cancer diagnosis and check-up tests, the low cut-off limit between prostate hyperplasia and $\mathrm{CaP}$ can cause inaccuracy in results. Nanotecnology gives us the opportunity to develop novel detection techniques with higher sensitivity, lower sample requirement and shorter analysis time on a miniaturized scale. Label based nanobiosensing introduces some unique imaging devices, such as fluorescent nanoparticles (quantum dots, europium, etc.) that have strong, long-lasting fluorescent activity. In addition, SERS nanoparticles can have sharper, more specific bands than fluorophores while being insensitive to humidity or oxygen. In addition, all these nanoparticles have size-dependant variety, allowing them to emit different wavelengths. Magnetic resonance signal sensitivity, low detection limits, low toxicity and excellent biocompatibility makes MNPs unique in cell tracking, MRI, bioseparation and tissue engineering. These nanoparticles can also be used in designing assays with low limit of detection and low antibody requirement. Moreover, label-free nanobiosensing systems like SPR, nanowirebased assays and microcantilevers make it possible to use unmodified samples, with the possibility of highly sensitive real-time measurement. As a good example, SWNT immunosensors are believed to be beneficial in designing novel bio-arrays for multiplexed detection of cancer biomarkers.

In summary, it is expected that nanotechnology will soon make it possible to have cancer test results available within minutes, resulting in reduced hospital or clinic visits, decreased costs and improved clinical outcomes. Finally, important issues such as reproducibility, specificity and cost per test need to be addressed for cancer diagnostic tests before nanotechnology-based platforms are introduced into clinical applications and commercialization.

\section{ACKNOWLEDGMENTS}

The author would like to thank Dr Seyed Javad Rasuli and Dr Ghazaleh EskandariSedighi for their help in manuscript preparation. 


\section{DECLARATIONS}

\section{Funding}

Not applicable.

\section{Ethics approvals and consent to participate}

Not applicable.

\section{Conflict of interest}

The author declares that there is no conflict of interest regarding publication of this article.

\section{REFERENCES}

1. Bray F, Ferlay J, Soerjomataram I, Siegel RL, Torre LA, Jemal A. Global cancer statistics 2018: GLOBOCAN estimates of incidence and mortality worldwide for 36 cancers in 185 countries. CA: A Cancer Journal for Clinicians. 2018;68(6):394-424.

2. Healy DA, Hayes CJ, Leonard P, McKenna L, O'Kennedy R. Biosensor developments: application to prostate-specific antigen detection. TRENDS in Biotechnology. 2007;25(3):125-31.

3. Mansoori GA, Mohazzabi P, McCormack P, Jabbari S. Nanotechnology in cancer prevention, detection and treatment: bright future lies ahead. World Review of Science, Technology and Sustainable Development. 2007;4(2-3):226-57.

4. Wang AZ, Gu FX, Farokhzad OC. Nanoparticles for cancer diagnosis and therapy. Safety of Nanoparticles: Springer; 2009. p. 209-35.

5. Jain K. Proteomics-based anticancer drug discovery and development. Technology in cancer research \& treatment. 2002;1(4):231-6.

6. Makarov DV, Loeb S, Getzenberg RH, Partin AW. Biomarkers for prostate cancer. Annual review of medicine. 2009;60:139-51.
7. Partin AW, Marks LS. Prostate-specific antigen and new serum biomarkers for evaluation of chemopreventive agents. Urology. 2001;57(4):132-6.

8. Schalken J. New developments in the pathobiology of prostate disease. european urology supplements. 2006;5(12):729-36.

9. You J, Cozzi P, Walsh B, Willcox M, Kearsley J, Russell P, et al. Innovative biomarkers for prostate cancer early diagnosis and progression. Critical reviews in oncology/hematology. 2010;73(1):10-22.

10. Yu X, Munge B, Patel V, Jensen G, Bhirde A, Gong JD, et al. Carbon nanotube amplification strategies for highly sensitive immunodetection of cancer biomarkers. Journal of the American Chemical Society. 2006;128(34):11199-205.

11. Yuhi T, Nagatani N, Endo T, Kerman K, Takata M, Konaka H, et al. Gold nanoparticle based immunochromatography using a resin modified micropipette tip for rapid and simple detection of human chorionic gonadotropin hormone and prostate-specific antigen. Science and Technology of Advanced Materials. 2006;7(3):276-81.

12. Sardana G, Diamandis EP. The kallikrein family of proteins as urinary biomarkers for the detection of prostate cancer. Clinical biochemistry. 2009;42(13):1483-6.

13. Garbis SD, Tyritzis SI, Roumeliotis T, Zerefos P, Giannopoulou EG, Vlahou A, et al. Search for potential markers for prostate cancer diagnosis, prognosis and treatment in clinical tissue specimens using amine-specific isobaric tagging (iTRAQ) with twodimensional liquid chromatography and tandem mass spectrometry. The Journal of Proteome Research. 2008;7(8):3146-58.

14. Zhou J, Huang L, Wang W, Pang J, Zou Y, Shuai X, et al. Prostate cancer targeted 
MRI nanoprobe based on superparamagnetic iron oxide and copolymer of poly (ethylene glycol) and polyethyleneimin. Chinese Science Bulletin. 2009;54(18):3137-46.

15. Wang AZ, Bagalkot V, Vasilliou CC, Gu F, Alexis F, Zhang L, et al. Superparamagnetic iron oxide nanoparticleaptamer bioconjugates for combined prostate cancer imaging and therapy. ChemMedChem. 2008;3(9):1311-5.

16. Azzazy HM, Mansour MM, Kazmierczak SC. From diagnostics to therapy: prospects of quantum dots. Clinical biochemistry. 2007;40(13):917-27.

17. Dong W, Guo L, Wang M, Xu S. CdTe QDs-based prostate-specific antigen probe for human prostate cancer cell imaging. Journal of Luminescence. 2009;129(9):926-30.

18. Gao X, Xing Y, Chung LW, Nie S. Quantum Dot Nanotechnology for Prostate Cancer Research. Prostate Cancer: Springer; 2007. p. 231-44.

19. Smith AM, Duan H, Mohs AM, Nie S. Bioconjugated quantum dots for in vivo molecular and cellular imaging. Advanced drug delivery reviews. 2008;60(11):1226-40.

20. Bagalkot V, Zhang L, Levy-Nissenbaum E, Jon S, Kantoff PW, Langer R, et al. Quantum dot-aptamer conjugates for synchronous cancer imaging, therapy, and sensing of drug delivery based on bifluorescence resonance energy transfer. Nano letters. 2007;7(10):3065-70.

21. Shi C, Zhu Y, Cerwinka WH, Zhau HE, Marshall FF, Simons JW, et al., editors. Quantum dots: emerging applications in urologic oncology. Urologic Oncology: Seminars and Original Investigations; 2008: Elsevier.
22. Shi C, Zhu Y, Xie Z, Qian W, Hsieh C-L, Nie $S$, et al. Visualizing human prostate cancer cells in mouse skeleton using bioconjugated near-infrared fluorescent quantum dots. Urology. 2009;74(2):446-51.

23. Härmä H, Soukka $T$, Lövgren $T$. Europium nanoparticles and time-resolved fluorescence for ultrasensitive detection of prostate-specific antigen. Clinical chemistry. 2001;47(3):561-8.

24. Huhtinen P, Soukka T, Lövgren T, Härmä H. Immunoassay of total prostate-specific antigen using europium (III) nanoparticle labels and streptavidin-biotin technology. Journal of immunological methods. 2004;294(1):111-22.

25. Ye Z, Tan M, Wang G, Yuan J. Preparation, characterization and application of fluorescent terbium complex-doped zirconia nanoparticles. Journal of fluorescence. 2005;15(4):499-505.

26. Hudson SD, Chumanov G. Bioanalytical applications of SERS (surface-enhanced Raman spectroscopy). Analytical and bioanalytical chemistry. 2009;394(3):679-86.

27. Yoon K-J, Seo H-K, Hwang H, Pyo D-J, Eom I-Y, Hahn J-H, et al. Bioanalytical application of SERS immunoassay for detection of prostate-specific antigen. Bulletin of the Korean Chemical Society. 2010;31(5):1215-8.

28. Park H-Y, Driskell JD, Kwarta KM, Lipert RJ, Porter MD, Schoen C, et al. Ultrasensitive immunoassays based on surface-enhanced Raman scattering by immunogold labels. Surface-Enhanced Raman Scattering: Springer; 2006. p. 427-46.

29. Grubisha DS, Lipert RJ, Park H-Y, Driskell J, Porter MD. Femtomolar detection of prostate-specific antigen: an immunoassay 
based on surface-enhanced Raman scattering and immunogold labels. Analytical chemistry. 2003;75(21):5936-43.

30. Schlücker S, Küstner B, Punge A, Bonfig R, Marx A, Ströbel P. Immuno-Raman microspectroscopy: In situ detection of antigens in tissue specimens by surface-enhanced Raman scattering. Journal of Raman Spectroscopy. 2006;37(7):719-21.

31. Sun C, Su K-H, Valentine J, Rosa-Bauza YT, Ellman JA, Elboudwarej O, et al. Timeresolved single-step protease activity quantification using nanoplasmonic resonator sensors. ACS nano. 2010;4(2):978-84.

32. Shubayev VI, Pisanic TR, Jin S. Magnetic nanoparticles for theragnostics. Advanced drug delivery reviews. 2009;61(6):467-77.

33. Kim KY. Nanotechnology platforms and physiological challenges for cancer therapeutics. Nanomedicine: Nanotechnology, Biology and Medicine. 2007;3(2):103-10.

34. Chanda N, Kan P, Watkinson LD, Shukla R, Zambre A, Carmack TL, et al. Radioactive gold nanoparticles in cancer therapy: therapeutic efficacy studies of GA-198 AuNP nanoconstruct in prostate tumor-bearing mice. Nanomedicine: Nanotechnology, Biology and Medicine. 2010;6(2):201-9.

35. Roa W, Zhang X, Guo L, Shaw A, Hu X, Xiong $\mathrm{Y}$, et al. Gold nanoparticle sensitize radiotherapy of prostate cancer cells by regulation of the cell cycle. Nanotechnology. 2009;20(37):375101.

36. Chen H, Jiang C, Yu C, Zhang S, Liu B, Kong J. Protein chips and nanomaterials for application in tumor marker immunoassays. Biosensors and Bioelectronics. 2009;24(12):3399-411.

37. Lee Y, Lee SH, Kim JS, Maruyama A, Chen X, Park TG. Controlled synthesis of
PEI-coated gold nanoparticles using reductive catechol chemistry for siRNA delivery. Journal of controlled release. 2011;155(1):310.

38. Thaxton CS, Elghanian R, Thomas AD, Stoeva SI, Lee J-S, Smith ND, et al. Nanoparticle-based bio-barcode assay redefines "undetectable" PSA and biochemical recurrence after radical prostatectomy. Proceedings of the National Academy of Sciences. 2009;106(44):1843742.

39. Xia N, Deng D, Wang Y, Fang C, Li S-J. Gold nanoparticle-based colorimetric method for the detection of prostate-specific antigen. International journal of nanomedicine. 2018;13:2521-30. PubMed PMID: 29731627. eng.

40. Fortina P, Wang J, Surrey S, Park JY, Kricka LJ. Beyond MicrotechnologyNanotechnology in Molecular Diagnosis. Integrated Biochips for DNA Analysis: Springer; 2007. p. 187-97.

41. Zheng G, Patolsky F, Cui Y, Wang WU, Lieber CM. Multiplexed electrical detection of cancer markers with nanowire sensor arrays. Nature biotechnology. 2005;23(10):1294-301.

42. Maehashi K, Matsumoto K. Label-free electrical detection using carbon nanotubebased biosensors. Sensors. 2009;9(7):536878.

43. Okuno J, Maehashi K, Kerman K, Takamura Y, Matsumoto K, Tamiya E. Labelfree immunosensor for prostate-specific antigen based on single-walled carbon nanotube array-modified microelectrodes. Biosensors and Bioelectronics. 2007;22(9):2377-81.

44. Quintero-Jaime AF, Berenguer-Murcia Á, Cazorla-Amorós D, Morallón E. Carbon 
Nanotubes Modified With $\mathrm{Au}$ for Electrochemical Detection of Prostate Specific Antigen: Effect of Au Nanoparticle Size Distribution. Frontiers in Chemistry. 2019 2019-March-27;7(147). English.

45. Kang B, Wang H, Lele T, Tseng Y, Ren $\mathrm{F}$, Pearton S, et al. Prostate specific antigen detection using AlGaN/GaN high electron mobility transistors. Applied physics letters. 2007;91(11):112106-.

46. Ahn J-H, Im M, Choi Y-K, editors. Labelfree electrical detection of PSA by a nanogap field effect transistor. Proc Micro Total Analysis Systems; 2008.

47. Kim J, Junkin M, Kim D-H, Kwon S, Shin YS, Wong PK, et al. Applications, techniques, and microfluidic interfacing for nanoscale biosensing. Microfluidics and Nanofluidics. 2009;7(2):149-67.

48. Su L-C, Chen R-C, Li Y-C, Chang Y-F, Lee Y-J, Lee C-C, et al. Detection of prostatespecific antigen with a paired surface plasma wave biosensor. Analytical chemistry. 2010;82(9):3714-8.

49. Hong Y, Huh Y-M, Yoon DS, Yang J. Nanobiosensors based on localized surface plasmon resonance for biomarker detection. Journal of Nanomaterials. 2012;2012:111.

50. Hwang WS, Sim SJ. A strategy for the ultrasensitive detection of cancer biomarkers based on the LSPR response of a single AuNP. Journal of nanoscience and nanotechnology. 2011;11(7):5651-6.

51. Unser S, Bruzas I, He J, Sagle L. Localized Surface Plasmon Resonance Biosensing: Current Challenges and Approaches. Sensors. 2015;15(7):15684-716.

52. Choi J-W, Oh B-K, Jang Y-H, Kang D-Y. Ultrasensitive immunoassay for prostate specific antigen using scanning tunneling microscopy-based electrical detection. Applied Physics Letters. 2008;93(3):033110.

53. Klein KM, Zheng J, Sitaraman S, Gewirtz A, Sarma D, Rajalakshmi S, editors. Array of Nano-Cantilevers as a Bio-Assay for Cancer Diagnosis. Electronic Components and Technology Conference; 2005: IEEE; 1999.

54. Vashist SK. A review of microcantilevers for sensing applications. J of Nanotechnology. 2007;3:1-18.

55. Hwang KS, Lee JH, Park J, Yoon DS, Park JH, Kim TS. In-situ quantitative analysis of a prostate-specific antigen (PSA) using a nanomechanical PZT cantilever. Lab on a Chip. 2004;4(6):547-52.

56. Wu G, Datar RH, Hansen KM, Thundat T, Cote RJ, Majumdar A. Bioassay of prostatespecific antigen (PSA) using microcantilevers. Nature biotechnology. 2001;19(9):856-60. 